\title{
Modified radical mastectomy for intraductal breast carcinoma in a patient with active pulmonary tuberculosis under thoracic epidural anaesthesia
}

\author{
Devi R U1 ${ }^{1}$, Kumar K R V², Aasthaa ${ }^{3}$, D.K Vinutha ${ }^{4}$, Kavyashree ${ }^{5}$ \\ ${ }^{1}$ Dr. Usha Devi R, Professor, ${ }^{2}$ Dr. Vasantha Kumar K R, Professor ${ }^{3}$ Dr Aasthaa, Junior Resident, ${ }^{4}$ Dr. Vinutha D. K, \\ Junior Resident, ${ }^{5}$ Dr. Kavyashree Junior Resident, All are affiliated with Adichunchingiri Institute of Medical Science, \\ Bellur, Mandya, Karnataka, India.
}

Address for correspondence: Dr. Kavyashree, Email: kavyashree.daanu@gmail.com

\begin{abstract}
The incidence of breast carcinoma is on the rise in Asian countries like India and pulmonary tuberculosis is one of the most common infectious diseases encountered here. Although coexistence of active pulmonary tuberculosis and breast cancer in same patient is rare, it poses a huge challenge to the anaesthetist when patients having the above conditions concomitantly are posted for surgeries like mastectomy. Here we report the anaesthetic management of a case of breast carcinoma with active pulmonary tuberculosis managed successfully under thoracic epidural anaesthesia chosen to avoid major hemodynamic, respiratory and other complications like operation theatre pollution and drug interactions between anti tubercular and anticancer drugs and drugs used for general anesthesia.
\end{abstract}

Keywords: Active pulmonary tuberculosis, Right Intra ductal breast carcinoma, Thoracic epidural anesthesia, Radical mastectomy

\section{Background}

Pulmonary tuberculosis is one of the most contagious bacterial infections that involve the lungs and it can spread to other organs easily. Such patients if given general anesthesia can have the risk of respiratory complications like severe cough, bronchospasm, massive airway hemorrhage, pnemothorax. There can also be severe hemodynamic changes, contamination of anaesthesia machine circuits and operation theatre as well as exposure of OT personnel to infection.

Tuberculosis is a common disease in India. Majority of patients have subclinical infection and about $10 \%$ of these people develop clinically detectable disease [1]. In the developing countries of Asia, the health care burden on account of breast cancer has been on the rise. Incidence of carcinoma of breast varies from 12 to 31 cases per 100,000 women [2]. Over 100,000 new breast cancer patients are estimated to be diagnosed annually in India. The age standardized incidence rates (AARs) range from 6.2 to 39.5 per 100,000 women [3].

Our patient presented with right Intraductal breast carcinoma and open pulmonary tuberculosis and was posted for modified radical mastectomy. We chose neuraxial blockade in the form of thoracic epidural anesthesia for our patient management.

\section{Case History}

45-year-old female patient was admitted for surgery with the chief complaint of a painless lump in the right breast since four months accompanied with a history of occasional dry cough, decreased appetite, and significant weight loss present over past one year. On examination her height and weight were $150 \mathrm{~cm}$ and $50 \mathrm{kgs}$, respectively. She had a lump in the upper inner quadrant of the right breast approximately $2 \mathrm{~cm}$ x $2 \mathrm{~cm}$ in size, diagnosed as intraductal carcinoma.

Manuscript received $26^{\text {th }}$ May 2016

Reviewed: $7^{\text {th }}$ June 2016

Author Corrected: $18^{\text {th }}$ June 2016

Accepted for Publication $29^{\text {th }}$ June 2016 
Ultrasound showed breast lump in right upper inner quadrant at 2 O-clock position and FNAC report confirmed ductal carcinoma.

Plain chest radiograph showed irregular cavitary lesion in the upper and lower lobes of the right lung with areas of nodular opacity and reticulo nodular lesions and X- ray thoraco- lumbar spine ruled out any metastasis to spinal column.

Sputum examination revealed presence of acid fast bacilli suggestive of pulmonary tuberculosis. Metastasis to chest wall or spine was ruled out.

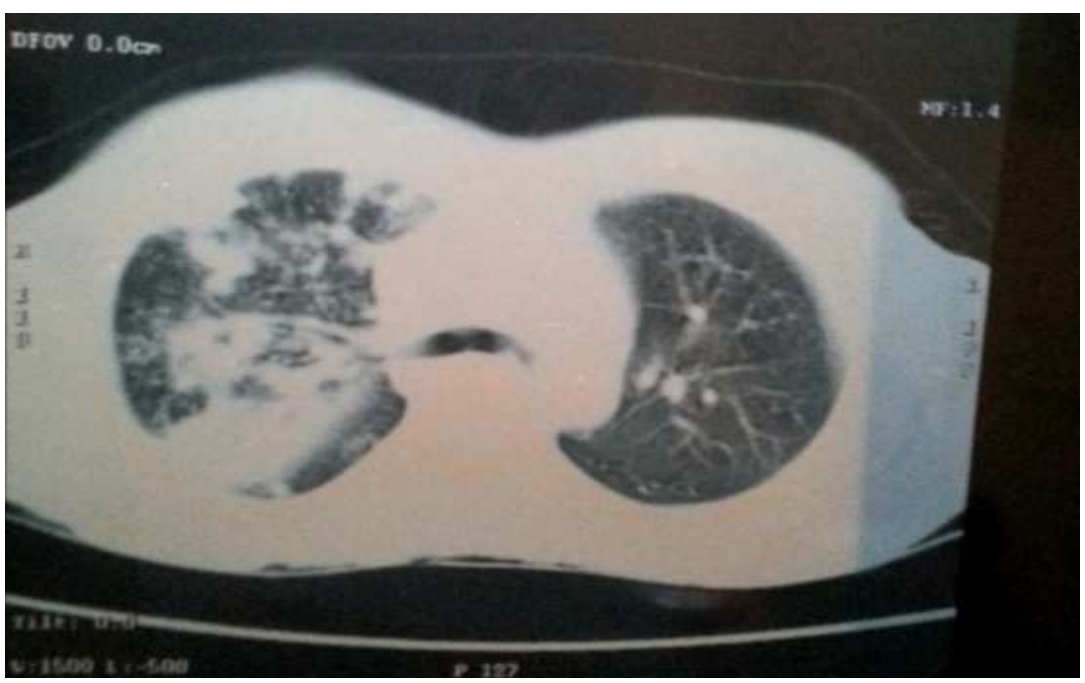

Figure 1: CT chest showed multi focal patchy areas of consolidation in right upper lobe and superior segment of right lower lobe with focal areas of thin walled cavitations. A "tree in bud" appearance was seen, suggestive likely of tuberculosis.

The preoperative pulmonary function test indicated mild obstruction. Echocardiography showed mitral valve prolapse but no mitral regurgitation with an ejection fraction of $60 \%$. No other special findings were detected in the electrocardiogram, and other blood tests. The patient had been put on anti tubercular medicines (rifampicin, streptomycin, Isoniazid) from 3 weeks before surgery. She was not on any chemotherapy or radiotherapy for breast carcinoma. She and been refused surgery earlier in view of the high anaesthesia risk.

Thoracic epidural anesthesia supplemented with IV Sedation was planned. An informed consent was taken from the patient and relatives after explaining the risks involved. She received preoperatively IV inj. midazolam $1 \mathrm{mg}$ and inj.ondansetron $4 \mathrm{mg}$. Under all aseptic precautions with the patient in the lateral decubitus position, after subcutaneous infiltration with $2 \%$ lignocaine with number 26 hypodermic needle, thoracic epidural space between T4- T5 vertebrae was identified by means of a loss of resistance technique. An 18 Gauge epidural catheter was inserted $4 \mathrm{~cm}$ into the epidural space through an epidural Touhy needle.

Catheter was fixed and a test dose of $3 \mathrm{ml}$ of $2 \%$ lignocaine with 1:200,000 epinephrine was given through the catheter to rule out intravascular or subarachnoid placement. Initially Inj. bupivacaine $0.5 \% 8 \mathrm{ml}$ with fentanyl (50 microgram) titrated incrementally to a total of $14 \mathrm{ml}$ was given to achieve surgical anesthesia. Sensory blockade was assessed by pin prick, achieved from the inferior border of clavicle to the inferior costal margin.

This was supplemented with IV sedation in the form of IV Midazolam 1mg, Inj. Propofol 50mg bolus followed by $100 \mathrm{mg}$ as infusion and inj. ketamine 100mg in divided doses. The patient also received infiltration of the axilla with $6 \mathrm{ml}$ of $2 \%$ lignocaine later on. The catheter was left in place and used for postoperative analgesia using $0.125 \%$ bupivacaine for 2 days. The surgery time was 80 minutes, total anesthesia time 100 minutes.

Surgery was completed without any hemodynamic disturbance. The patient was shifted to recovery room and later to surgical ICU for post operative management. She was comfortable and pain free postoperatively. 


\section{Discussion}

Pulmonary tuberculosis (TB) is a contagious bacterial infection that involves the lungs. It may spread to other organs. Pulmonary tuberculosis (TB) is caused by the bacterium Mycobacterium tuberculosis (M. tuberculosis). TB is contagious.

Certain conditions can increase the risk of tuberculosis e.g. prolonged corticosteroid treatment, gastrectomy, leukemia, silicosis, Hodgkin's disease, pneumoconiosis, severe or poorly controlled diabetes, pregnancy and children with measles or whooping cough. [4]

The issues of importance to the anaesthetist in a tuberculous patient are as follows:

1. The general state of the patient's health and the effect of the disease on organ function are of concern. There is also the possibility of coexistence of HIV.

2. There is a risk of massive airway hemorrhage during the critical periods of tracheal intubation or extubation and/or careless airway suction [5]. Pulmonary Tuberculosis can cause massive hemoptysis through multiple mechanisms. [6] This could occur because of active cavitatory or non cavitatory lung disease, sudden rupture of a Rasmussen's aneurysm, residual bronchiectasis or erosion of a broncholith through a vessel and into an airway.

3. Bronchial stimulation leading to severe cough can result in pneumothorax or bronchospasm. The hemodynamic changes during insertion or removal of endotracheal tube may be severe.

4. The treatment that the patient is taking may have the potential for drug interactions.

- Pharmacokinetic changes following the induction of liver enzymes may cause drug interactions. Rifampicin is the major cause for most of such drug interactions. It is a potent inducer of the cytochrome P450 system especially of isoenzyme 3A4. This can result in increased metabolism with sub therapeutic effects or an increased production of toxic metabolites. [7] High doses of the drug can cause thrombocytopenia and reduce the effect of nonsteroidal anti-inflammatory agents like diclofenac. Patients on anti tuberculosis therapy may be at increased risk of halothane hepatitis.
Increased metabolism may be important in total intravenous anaesthesia and may result in greater potential for intraoperative awareness [7].

- Fentanyl and alfentanil are both extensively metabolised by CYP450 3A4 and hence show a potential for shortened duration of action. [7] A loss of analgesic effect of oral morphine has been demonstrated following pretreatment with rifampicin. [8] Analgesia should therefore be titrated to effect as the patient may require more frequent dosing. Oral midazolam may be ineffective with rifampicin [7].

- Streptomycin is an aminoglycoside antibiotic possessing neuromuscular blocking activity. The neuromuscular blockade produced by it is only reversed partially by neostigmine. This extensive potentiating of the neuromuscular effects of relaxants by antibiotics can be due to the fact that antibiotics not only have a curare-like stabilizing effect on the post junctional membrane but also decrease presynaptic acetylcholine release. [9] Streptomycin is potentially capable of interacting with non-depolarizing muscle relaxant drugs like vecuronium resulting in respiratory depression and/or prolonged apnea. [7, 9] INH (a CYP 450 inhibitor) can cause sensory neuropathy. [7]

5. Tuberculosis is an airborne disease. The risk of transmission of tuberculosis to the staff and other patients, especially in a setup with lack of facility for proper scavenging is of serious concern. Our patient was last on the surgical list of the day to facilitate theatre decontamination. Everyone associated with her in the theatre wore an efficient well fitting mask.

Belzarena SD in a comparative study between single shot epidural block and general anaesthesia for oncologic mastectomy in 40 patients concluded that the former technique using a local anaesthetic with an opioid is an adequate option for mastectomy [10]. Although epidural anesthesia and analgesia also have complications which include failure to produce anesthesia on the first attempt (4.1\%), bleeding/ intravascular cannulation $(0.67 \%)$, unintentional dural puncture $(0.61 \%)$, paraesthesia $(0.16 \%)$, and local anesthetic toxicity $(0.12 \%)$, [11] we chose neuroxial (thoracic epidural) anesthesia in our patient to avoid interference with patients airway and postoperative 
nausea and vomiting. There was a lower cost of procedure and duration of hospital stay. Other authors have commented upon significantly lower chances of airway hemorrhage or aspiration; lack of significant changes in respiratory function; reduction in stress response and reduction in opiate use. [12]

Though it did not occur in our patient, it should be kept in mind that a high level of blockade can give rise to hypotension or bradycardia [10]. Immediate postoperative pain was effectively controlled by injection of opioid through the epidural catheter in our patient. Epidural anaesthesia is said to reduce the risk of recurrence or metastasis during the initial years of follow up of patients probably because of the preservation of immune mechanisms [13].

Sole thoracic epidural anesthesia (TEA) has been used earlier for bilateral mammoplasty [14] and with brachial plexus block for modified radical mastectomy (MRM) [15]. Studies have shown the usefulness of the technique to reduce the need for prolonged ventilation or reintubation; improved lung function and blood oxygenation. There is a reduction in pulmonary complications due to early ambulation [16].

Para vertebral block has been used to perform major operations for breast cancer with minimal complications and a low rate of conversion to general anesthesia. It markedly improves the quality of recovery after breast cancer surgery and provides the patient with the option of ambulatory discharge [17].

Thoracic epidural anesthesia is a relatively difficult technique and requires an experienced anaesthetist. Care should be exercised not to involve the phrenic nerve or cause Horner's syndrome, dural puncture or neurological injury.

Conclusion: Thoracic epidural technique was used effectively in our patient for radical mastectomy.

It provided excellent conditions for surgery intraoperatively and efficient analgesia in the postoperative period without complications. It reduced opioid usage and improved acceptance of chest physiotherapy by the patient.

Hence it is an excellent anesthesia technique for modified radical mastectomy in select group of patients with breast carcinoma with concomitant open pulmonary tuberculosis.
Funding: Nil, Conflict of interest: None initiated, Permission from IRB: Yes

\section{References}

1. G S Sainani Ed. Pulmonary Tuberculosis in API text book of Medicine. 1999; 241-245.

2. Asegaonkar BN, Zine SR, Takalkar UV, Kulkarni U, Asegaonkar SB, Kodlikeri P. Thoracic epidural anesthesia for modified radical mastectomy in carcinoma of breast patient with chronic obstructive pulmonary disease: A case report. International Journal of Case Reports and Images 2013;4(5):546-550.

3. Gaurav Agarwal and Pooja Ramakant. Breast Cancer Care in India: The Current Scenario and the Challenges for the Future. Breast Care (Basel). 2008 Mar; 3(1): 21-27.Published online 2008 Feb 22. doi: 10.1159/000115288 PMCID: PMC2931014

4. Kamboj M, Sepkowitz KA The risk of tuberculosis in patients with cancer. Clinical Infect Dis jun 1; 42(11): 1592-1595. Epub 2006 Apr 27 PMID-16652317.

5. Huh J, Kang SS. Bronchial bleeding induced by suction in a patient with bronchial anthracofibrosis. Korean J Anesthesiol. 2004; 46:624-627.

6.http://critical.med.ualberta.ca/FileArchive/ /media/cri ticalcare/Documents/Causesandmanagementofmassiveh emoptysis.pdf.

7. Jackson TA, Thomas JM, South Afr .Tuberculosis: the implications for anaesthesia J Anaesth Analg 2013; 19(6):301-305.

8. Fromm MF, Eckhardt K, Schanzle G, et al. Loss of analgesic effect of morphine due to coadministration of rifampicin. Pain 1997; 72(1-2):261-267.

9. Burkett L, Bikhazi GB, Thomas KC Jr, et al. Mutual potentiation of the neuromuscular effects of antibiotics and relaxants. Anesth Analg. 1979 Mar-Apr; 58 (2):107-15.

10. Belzarena SD Comparative study between thoracic epidural block and general anesthesia for oncologic mastectomy. Rev Bras Anestesiol. 2008 NovDec;58(6):561-8.

11. Tanaka K, Watanabe R, Harada T, Dan K. 
Extensive application of epidural anesthesia and analgesia in a university hospital: incidence of complications related to technique. Reg Anesth. 1993 Jan-Feb;18(1):34-8.

12. E P Lynch, K J Welch, J M Carabuena, and T J Eberlein. Thoracic epidural anesthesia improves outcome after breast surgery Ann Surg. 1995 Nov; 222(5): 663-669.

13. Exadaktylos AK, Buggy DJ, Moriarty DC, et al. Can anesthetic technique for primary breast cancer surgery affect recurrence or metastasis? Anesthesiology 2006; 105:660-4. PMID - 17006061.

14. O'Connor PJ, Moysa GL, Finucane BT. Thoracic epidural anesthesia for bilateral reduction mammoplasty in a patient with Klippel-Feil syndrome. Anesth Analg 2001; 92(2):514-6.PMID-11159260.
15. Sundarathiti $\mathrm{P}$, Pasutharnchat $\mathrm{K}$, Kongdan $\mathrm{Y}$, Suranutkarin PE. Thoracic epidural anesthesia (TEA) with $0.2 \%$ ropivacaine in combination with ipsilateral brachial plexus Block (BPB) for Modified Radical Mastectomy (MRM). J Med Assoc Thai 2005; 88(4):513-20.PMID -16146257.

16. Popping DM, Elia N, Marret E, Remy C, Tramer MR. Protective effects of epidural analgesia on pulmonary complications after abdominal and thoracic surgery: a meta-analysis. Arch Surg 2008 Oct; 143 (10):990-9.PMID-18936379.

17. E Coveney, C R Weltz, R Greengrass, J D Iglehart, et al. Use of paravertebral block anesthesia in the surgical management of breast cancer: experience in 156 cases. Ann Surg.1998 Apr; 227(4); 496-501.PMID9563536.

\section{How to cite this article?}

Devi R U, Kumar K R V, Aasthaa, D.K Vinutha, Kavyashree. Modified radical mastectomy for intraductal breast carcinoma in a patient with active pulmonary tuberculosis under thoracic epidural anaesthesia. Int J Med Res Rev 2016;4 (7):1216-1220.doi:10.17511/ijmrr.2016.i07.24. 